\title{
The MRI characteristics of the no-flow region are similar in reperfused and non- reperfused myocardial infarcts: an MRI and histopathology study in swine
}

Gabriel A. Elgavish ${ }^{1,2,3}$, Tamas Simor ${ }^{1,3,4}$, Rob J. van der Geest ${ }^{5}$, Pal Suranyi ${ }^{6}$, Pal P. Kiss ${ }^{3}$, Zsofia Lenkey ${ }^{1,3,4}$, Robert Kirschner ${ }^{1,3}$, Dezhi Wang ${ }^{7}$, Brigitta C. Brott ${ }^{2}$ and Akos Varga-Szemes ${ }^{1,6^{*}}$ (D)

\begin{abstract}
Background: The no-flow region (NF) visualised by magnetic resonance imaging (MRI) in myocardial infarction (MI) has been explained as the product of reperfusion-injury-induced microvascular obstruction. However, a similar MRI phenomenon occurs in non-reperfused MI. Accordingly, our purpose was to compare the MRI and histopathologic characteristics of the NF in reperfused and non-reperfused Mls.

Methods: Reperfused $(n=7)$ and non-reperfused Mls $(n=7)$ were generated in swine by percutaneous balloon occlusion and microsphere embolisation techniques. Four days post-Ml, animals underwent myocardial T2-mapping, early and serial late gadolinium enhancement MRI. MI and NF were compared between the models using the independent samples $t$ test. Serial measurements were analysed using repeated measures analysis of variance. Triphenyltetrazolium chloride (TTC) macroscopic and microscopic histopathologic assessment was also performed.

Results: The Ml size in the reperfused and non-reperfused groups was $17.1 \pm 3.4 \mathrm{ml}$ and $19.4 \pm 8.1 \mathrm{ml}$, respectively ( $p=0.090)$, in agreement with $\Pi C$ assessment $(p=0.216 ; p=0.484)$, and the NF size was $7.7 \pm 2.4 \mathrm{ml}$ and $8.1 \pm 1$. $9 \mathrm{ml}$, respectively $(P=0.211)$. Compared to the reference 2 -min post-contrast measurement, the NF size was significantly reduced at $20 \mathrm{~min}$ in the reperfused group and at $25 \mathrm{~min}$ in the non-reperfused group (both $p<0.001)$. Nevertheless, the NF was still detectable at $45 \mathrm{~min}$ after injection. No significant T2 difference was observed between the groups ( $p>0.326$ ). Histopathologic assessment revealed extensive calcification and hemosiderin deposition in the NF of the reperfused Ml, but not in the non-reperfused MI.

Conclusions: The NF in non-reperfused and reperfused Mls have similar characteristics on MRI despite the different pathophysiologic and underlying histopathologic conditions, indicating that the presence of the NF alone cannot differentiate between these two types of Ml.
\end{abstract}

Keywords: Myocardial infarction, Microvascular obstruction, No-flow region, Magnetic resonance imaging (MRI), Late gadolinium enhancement

\footnotetext{
* Correspondence: vargaasz@musc.edu

'Department of Biochemistry and Molecular Genetics, University of Alabama

at Birmingham, MCLM 556, Birmingham, AL 35294-0005, USA

${ }^{6}$ Division of Cardiovascular Imaging, Department of Radiology and

Radiological Science, Medical University of South Carolina, 25 Courtenay Dr,

Charleston, SC 29425, USA

Full list of author information is available at the end of the article
} 


\section{Key points}

- The no-flow region (NF) is characteristic of both reperfused and non-reperfused myocardial infarct (MI)

- The size of the NF is significantly reduced in the post-contrast period

- The relative size of the NF and the uptake of contrast in the NF are similar in both models

- There is excessive calcification and haemorrhage in the NF in reperfused MI, but not in non-reperfused MI

\section{Background}

The clinical management of myocardial infarction (MI) by early reperfusion therapy aims to reduce the extent of the ischaemic injury and to prevent the long-term consequences of MI [1]. However, Braunwald and Kloner assert that reperfusion may also result in reperfusion injury, i.e., the death of potentially salvageable myocardium $[1,2]$. Reperfusion injury has been defined as reperfusion-related expansion of ischaemic cardiac injury resulting in decreased contractility, increased arrhythmogenic effects, irreversible conversion of reversible myocyte injury, and microvascular obstruction (MO) $[1,3]$.

The presence of $\mathrm{MO}$ seems to play a major role in longterm outcome after reperfusion post MI [4-11]. MO has been described as the strongest predictor of increase over time in left ventricle (LV) end-diastolic and end-systolic volumes post MI [4]. Patients with MO have been shown to have a significantly larger MI than patients without MO [5]. MO detected one week after the onset of MI has been suggested as an independent predictor of the size of MI at one year follow up and was found to be associated with adverse infarct healing, adverse LV remodelling, increased LV volumes, and lower ejection fractions [10]. Patients with MO have been reported as having more cardiovascular events than those without it [12]. Even independently of MI size, the presence of MO may remain a prognostic marker of post-MI complications [11]. Thus, as mentioned previously, the presence and/or the size of the $\mathrm{MO}$ might be one of the strong prognostic parameters after reperfusion post MI [4, 5, 8-11, 13].

MO has been defined as a product of reperfusion injury [1]. It has been shown, however, that the very same magnetic resonance imaging (MRI) phenomenon, i.e. central hypo-enhancement within the infarcted area, also occurs in MI without reperfusion [14, 15]. In non-reperfused MI, with no possibility of reperfusion injury, the underlying pathophysiologic condition of the similar MRI phenomenon might be different. Thus, to prevent any confusion when referring to the unenhanced central area within the MI observed by MRI, we use the term "no-flow region" (NF) instead of "microvascular obstruction". In this study, we aimed to compare the MRI and histopathologic characteristics of the NF in non-reperfused and reperfused MI.

\section{Methods}

Animal models

The study protocol was approved by the Institutional Animal Care and Use Committee and complied with the Guidelines for the Care and Use of Laboratory Animals (National Institutes of Health). Male swine $(n=14$, weight $24 \pm 3.1 \mathrm{~kg}$ ) were anesthetised with an intramuscularly administered mixture of telazol $(4.4 \mathrm{mg} / \mathrm{kg})$ and xylazine $(4.4 \mathrm{mg} / \mathrm{kg})$. Following intubation, animals were ventilated mechanically (Model 2000, Hallowell EMC, Pittsfield MA, USA) and anaesthesia was maintained by continuous administration of isoflurane $(2.0-2.5 \% \mathrm{~V} / \mathrm{V})$. Normal body temperature was supported using a heating pad. Heart rate and blood oxygen saturation were monitored, and electrocardiogram was recorded.

The right femoral artery was surgically prepared and cannulated using a 6-F arterial sheath (Pinnacle, Terumo Medical Co, Elkton, MD, USA). Heparin (100 IU/kg) was administered intravenously and the activated clotting time was monitored and adjusted as needed with additional heparin to maintain the activated clotting time above 300 s. A 6-F coronary guide catheter (RunWay Kimny Mini, Boston Scientific, Natick MA, USA) was introduced to cannulate the ostium of the left main coronary artery, and initial coronary angiography (Philips BV Pulsera, Best, The Netherlands) was performed.

\section{Reperfused myocardial infarct model}

In animals assigned to the reperfused group $(n=7)$, a 2.0-2.5 mm angioplasty balloon (Maverick, Boston Scientific, Natick MA, USA) was introduced over a coronary guide wire into the left circumflex (LCX) coronary artery. After determining the proper balloon position for the occlusion, the balloon catheter was inflated and left in position for 90 min to induce MI. After 90 min of ischaemia the balloon was deflated and removed, and coronary angiography was repeated to confirm recanalisation. The femoral artery was decannulated and surgically ligated, and the wound was closed.

\section{Non-reperfused myocardial infarct model}

In animals assigned to the non-reperfused group $(n=7)$, the microsphere embolisation technique was used [15]. Briefly, a 2.9-F straight tip microcatheter (Merit Maestro, Merit Medical, South Jordan UT, USA) was introduced over a coronary guide wire into the LCX coronary artery. After determining the proper microcatheter position for the occlusion, using the radiopaque tip of the microcatheter, the coronary guide wire was pulled out and a mixture of 900- $\mu \mathrm{m}$ microspheres (Embozene, CeloNova BioSciences, Inc., Newnan GA, USA) was flushed into the coronary artery under fluoroscopic control. When the lumen of the coronary artery distal to the tip of the microcatheter was completely filled with microspheres, 
the injection was stopped, the occlusion was confirmed by repeated angiography, and the microcatheter was removed. At the end of a 90-min monitoring period, the occlusion was confirmed by repeated angiography, and the femoral artery was decannulated and surgically ligated, and the wound was closed.

\section{Magnetic resonance imaging}

MRI studies were carried out four days after the induction of MI, using a 1.5 T GE Signa-Horizon CV/i scanner (GE Healthcare, Milwaukee, WI) equipped with a cardiac phased-array coil. Pigs were anaesthetised and ventilated mechanically as described previously. Imaging was performed during "breath-hold" at end-inspiration using the following parameters: field of view $=300 \mathrm{~mm}$, image-matrix $=256^{2}$, and slice thickness $=10 \mathrm{~mm}$. The flowchart of the MRI protocol is shown in Fig. 1.

\section{T2 mapping}

Following the pilot scans, short-axis oriented T2 mapping was carried out to study the T2 characteristics, especially the presence of myocardial haemorrhage in the MI. T2 maps were generated employing a double inversion recovery (IR), fast spin-echo pulse sequence using the following parameters: flip angle $(\alpha)=90^{\circ}$, echo train length $=24$, and echo time $(\mathrm{TE})=12-105 \mathrm{~ms}(12,20,30,45,60,75,90$, and $105 \mathrm{~ms}$ ). Images were collected at end-diastole in every second cardiac cycle.

\section{Early and late gadolinium enhancement imaging}

Following T2 mapping, a bolus of $0.2 \mathrm{mmol} / \mathrm{kg}$ gadopentetate dimeglumine (Magnevist, Bayer HealthCare Pharmaceuticals Inc, Wayne NJ, USA) was administered as the contrast agent (CA) for early gadolinium enhancement (EGE) and late gadolinium enhancement (LGE) imaging. Segmented, $180^{\circ}$-prepared, IR fast-gradientecho, short-axis (6 to 8 short-axis slices to cover the entire LV from apex to base) and long-axis (two-chamber, four-chamber, and LV outflow tract) oriented images were generated 2 min after CA administration (EGE imaging), and the acquisition was repeated at $10 \mathrm{~min}$ and thereafter every $5 \mathrm{~min}$ ending at $45 \mathrm{~min}$ (totaling eight LGE acquisitions in each animal). Imaging parameters were: $\alpha=25^{\circ}, \mathrm{TE}=3.2 \mathrm{~ms}$, views per segment $=16$, and repetition time $(\mathrm{TR})=5.5 \mathrm{~ms}$. The inversion time $(\mathrm{TI})$ for
EGE images was $500 \mathrm{~ms}$. For the LGE images, the applicable TI was set and continuously adjusted to an optimum value for nulling the signal of the healthy myocardium.

At the end of the planned in vivo MRI session, the animals were killed using a mixture of pentobarbital sodium, propylene glycol and ethyl alcohol $(0.2 \mathrm{ml} / \mathrm{kg})$ (Fatal-Plus, Vortech Pharmaceuticals, Dearborn MI, USA). Euthanasia was ascertained by electrocardiogram and auscultation above the thorax. The hearts were excised after euthanasia, rinsed with saline, and prepared for further studies.

\section{Magnetic resonance image analysis}

The Research Mass cardiovascular MR evaluation software (Leiden University Medical Center, Leiden, The Netherlands) was used for image analysis. The endocardial and epicardial contours of the LV were traced manually in every series of the short-axis images to delineate the myocardial area. Myocardial pixels were counted, and based on the pixel dimensions, the myocardial volume of each slice was determined, from which the total LV myocardial mass (LVM, g) was also calculated using the specific gravity of $1.05 \mathrm{~g} / \mathrm{ml}$ of myocardial tissue. To avoid observer bias, instead of manual contouring, a thresholding technique was used to delineate the MI and NF.

The mean signal intensity (SI) of the normal myocardium was measured using a region of interest containing at least 100 pixels. The mean SI of the remote myocardium plus five times the standard deviation (SD) of this mean was used as a threshold to delineate the pixels within the infarct ("infarcted pixels") (Fig. 2) [16, 17]. Pixels with SI over this threshold value were considered "MI pixels".

As the NF has been defined as a hypoperfused, unenhanced core within the highlighted MI, the SI of the NF is equal to or less than the SI of the remote myocardium. To overcome the problem of the NF not showing enhancement in EGE (the time period when its size is the largest), the size of the NF was measured indirectly. After MI thresholding, the unenhanced area of the NF was included in the MI area using the "MVO" contour function of the Mass application (Fig. 2c). Combination of the highlighted MI area and the unenhanced NF area

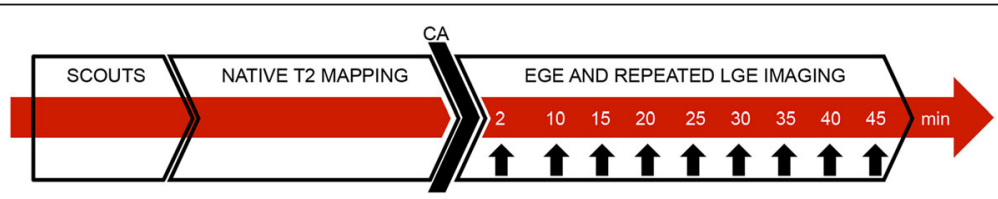

Fig. 1 Flowchart of the protocol for magnetic resonance imaging (MRI). Vertical black arrows indicate early gadolinium enhancement (EGE) and late gadolinium enhancement $(L G E)$ acquisitions. CA, contrast agent 

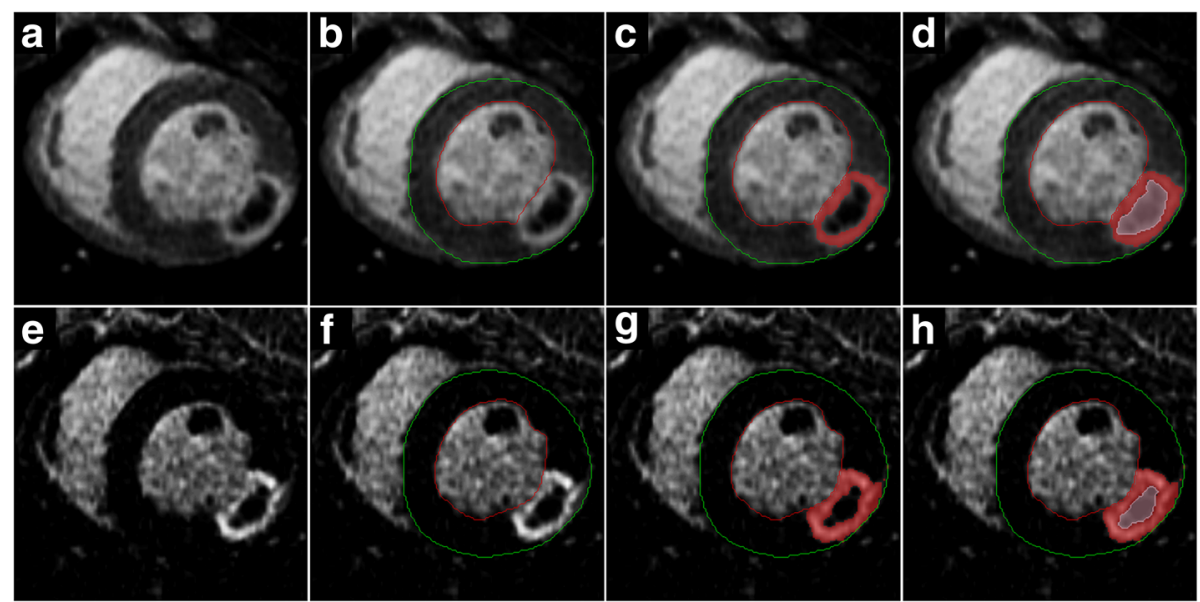

Fig. 2 Myocardial infarct (MI) and no-flow region (NF) segmentation. Short-axis inversion recovery (IR) early gadolinium enhancement (EGE) (a-d) and late gadolinium enhancement (LGE) (e-h) images obtained from an animal that underwent induction of non-reperfused Ml. The presence of the central NF surrounded by the rim of the Ml is apparent in the raw images (a, e). After the endocardial (red) and epicardial (green) contours were traced $(\mathbf{b}, \mathbf{f})$, the enhanced area was determined by the thresholding method using remote mean +5 SD as the cutoff $(\mathbf{c}, \mathbf{g})$. To measure the NF, the central non-enhanced area was traced using a dedicated contour $(\mathbf{d}, \mathbf{h})$. The total Ml size was calculated as the sum of the enhanced and the NF areas. The NF size was measured on the EGE image (d) and the total Ml size was assessed in the corresponding LGE image (h)

showed the true, total MI area. The difference between the total and the highlighted areas provided the NF area (Fig. 2d). The volume of the MI and NF were each expressed as a percentage of the LVM: the MI fraction (MIF) was calculated as the MI volume/LVM \% and the NF fraction (NFF) was calculated as the NF volume/ LVM \%. NF was also expressed as a percentage of the volume of the MI. Change in the size of the NF over time during the post-contrast period was analysed using the aforementioned technique. The actual size of the NF area was determined at each post-contrast time point.

\section{T2 calculation}

T2 was calculated from the TE dependence of the SI by means of a two-parameter, least squares, curve-fitting routine, using the following formula:

$$
\mathrm{SI}=\mathrm{SI}_{0} \times \mathrm{e}^{\left(-\mathrm{TE} \times \frac{1}{\mathrm{~T} 2}\right)}
$$

where $\mathrm{SI}_{0}$ is the signal intensity at the theoretical $\mathrm{TE}=0$ time point and it also represents the maximum SI.

The segmentation of the MI was carried out based on the areas determined in the EGE images. The T2 of the remote, infarcted (showing LGE), and the NF myocardium were obtained.

\section{Histopathologic assessment \\ Triphenyltetrazolium-chloride staining}

Triphenyltetrazolium-chloride (TTC) staining was used as a post-mortem reference standard to confirm the existence and the size of the MI. The hearts were breadsliced using a commercial meat slicer, and subsequently the slices were incubated for $20 \mathrm{~min}$ with a buffered (pH 7.4) $1.5 \%$ TTC solution at $37{ }^{\circ} \mathrm{C}$, similar to the method as described by Fishbein et al. [18]. After staining, the slices were immersed in $10 \%$ formalin for $20 \mathrm{~min}$ to increase the contrast between the healthy and the infarcted myocardium. Finally, both surfaces of each slice were digitally scanned with an image scanner, and both sides of each slice were analysed using ImageJ (Wayne Rasband, NIH, USA). The LV myocardium and the MI area were manually contoured and quantified. LVM and MIF were determined in each heart.

\section{Microscopic histologic assessment}

After TTC, whole-heart slices, including the defined epicardial and endocardial borders, were submitted for histopathologic assessment. The samples were fixed in $10 \%$ formalin, embedded in paraffin, and sectioned at 5- $\mu \mathrm{m}$ thickness. Haematoxylin-eosin staining (for general evaluation), Prussian blue staining (for haemorrhage detection), and Von Kossa staining (for calcium detection) were performed. Histologic samples were evaluated using a histomorphometry system (BioQuant Image Analysis, Nashville TN, USA) equipped with an Olympus BX-51 video microscope (Olympus America, Center Valley PA, USA).

\section{Statistical analysis}

Statistical analysis of the data obtained was carried out using MedCalc 13.2.2 (MedCalc Software, Ostend, Belgium). The Kolmogorov-Smirnov test was used to confirm that the data had a Gaussian distribution. T2 measurements and infarct-related parameters (MI and 
NF volume, MIF, and NFF) were compared in the reperfused and non-reperfused models using the independent samples $t$ test, and MI size and LVM measured by MRI and TTC were compared using the paired samples $t$ test. The size of the NF area was measured at each postcontrast time point and repeated measures analysis of variance was used to determine the time when the size of the NF was significantly different from the reference EGE measurement. Results were reported as mean \pm standard deviation (SD), unless otherwise noted. $p$ values lower than 0.050 were considered significant.

\section{Results}

All animals included in this study survived until day 4 and underwent MRI. The NF was detected in all 14 swine involved in this study. Representative long-axis EGE images (performed 2 min after CA administration) and LGE images (performed 15 min after CA administration) from both experimental groups are shown in Fig. 3.

LVM, MI, and NF measurements obtained by MRI and TTC are shown in Table 1. There was good agreement between MRI and TTC for the measurement of average LVM in each model (all $p>0.077$ ). There were no significant differences in mean MI size measured by MRI versus TTC, either in the reperfused group $(p=0.216)$, or in the non-reperfused group $(p=0.484)$. Furthermore, there was no significant difference in the mean MI size in the reperfused and nonreperfused groups $(p=0.090)$. The mean NF size, and its values normalised as a percentage of MI or LVM measured by EGE MRI, was not significantly different in the two experimental groups (all $p>0.114$ ).

$\mathrm{T} 2$ relaxation times measured in the remote, $\mathrm{MI}$, and NF area are reported in Table 2. No significant difference was observed between the groups, or between the remote and the NF area. T2 was significantly increased in the infarct area, i.e., the area showing LGE at $15 \mathrm{~min}$ post $\mathrm{CA}$ injection, in comparison to both the remote and NF areas (all $P<0.001$ ).

The volume of the NF gradually decreased with time after CA administration in both models (Fig. 4). NF measurements taken at and after $20 \mathrm{~min}$ were significantly different from the size of NF obtained in EGE in the reperfused model $(P<0.001)$, and there was a similar, significant reduction in the NF at $25 \mathrm{~min}$ in
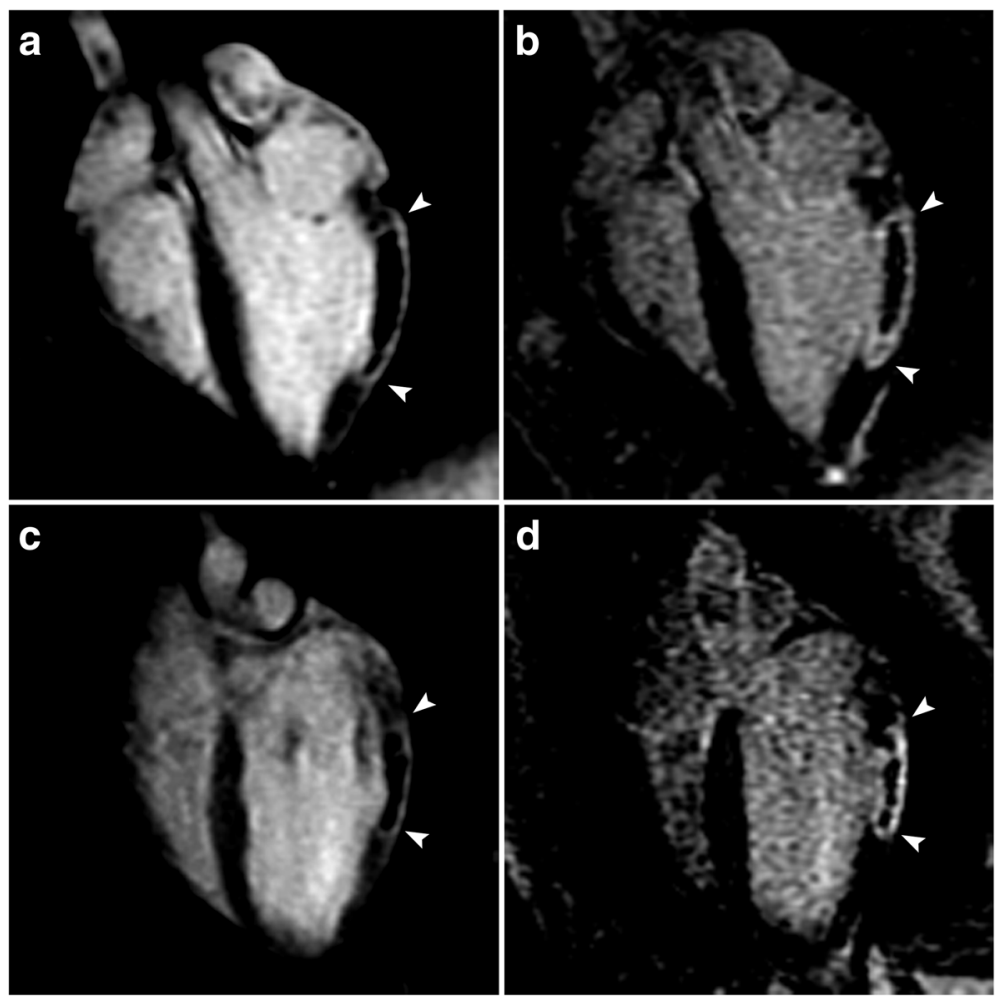

Fig. 3 Magnetic resonance imaging (MRI) appearance of myocardial infarct (MI) with a significant no-flow region (NF) at post-contrast time points that are used clinically. Representative long-axis early gadolinium enhancement (EGE) (2 min post-contrast) (a, c) and late gadolinium enhancement (LGE) (15 min post-contrast) $(\mathbf{b}, \mathbf{d})$ images obtained from animals with reperfused $\mathrm{MI}(\mathbf{a}, \mathbf{b})$ (top row) and non-reperfused (c, d) (bottom row). Mls are bracketed by arrowheads. The NF can be clearly visualised as a central unenhanced area within the enhanced rim of the MI, even in the late phase in both models 
Table 1 Average LVM, MI size (including NF) and NF size, as well as their normalised values (mean \pm SD) obtained by MRI and TTC

\begin{tabular}{|c|c|c|c|c|c|c|c|}
\hline \multirow[t]{2}{*}{ Parameter } & \multicolumn{3}{|l|}{ Reperfused } & \multicolumn{3}{|c|}{ Non-Reperfused } & \multirow[t]{2}{*}{$P^{*}$} \\
\hline & MRI & $\pi \mathrm{TC}$ & $P$ & MRI & $\pi \mathrm{TC}$ & $P$ & \\
\hline LVM (g) & $69.4 \pm 21.3$ & $74.2 \pm 27.9$ & 0.094 & $80.2 \pm 22.3$ & $76.6 \pm 21.7$ & 0.109 & 0.077 \\
\hline $\mathrm{Ml}(\mathrm{ml})$ & $17.1 \pm 3.4$ & $18.8 \pm 4.4$ & 0.216 & $19.4 \pm 8.1$ & $18.1 \pm 7.8$ & 0.484 & 0.090 \\
\hline MIF (\%LVM) & $27.3 \pm 12.2$ & $30.5 \pm 15.5$ & 0.202 & $24.3 \pm 7.7$ & $24.6 \pm 10.1$ & 0.625 & 0.158 \\
\hline $\mathrm{NF}(\mathrm{ml})$ & $7.7 \pm 2.4$ & & & $8.1 \pm 1.9$ & & & 0.211 \\
\hline NFF (\%LVM) & $12.6 \pm 7.8$ & & & $10.1 \pm 3.3$ & & & 0.114 \\
\hline NFF (\%MI) & $44.6 \pm 7.6$ & & & $41.5 \pm 9.9$ & & & 0.278 \\
\hline
\end{tabular}

MRI magnetic resonance imaging, TTC triphenyltetrazolium chloride staining, LVM left ventricular mass, MI myocardial infarct, MIF MI fraction, NF no-flow region, NFF no-flow fraction. ${ }^{*} P$ value for comparison of measurements in the reperfused and non-reperfused groups based on MRI

the non-reperfused model $(P<0.001)$. The NF was still observed in both models at 45 min after CA administration. The size of the NF, however, was larger in the nonreperfused group $(3.05 \pm 1.20$ versus $1.82 \pm 0.91 \mathrm{ml}$, respectively, $p<0.001$ ), indicating different $\mathrm{CA}$ uptake kinetics in the two models. The size of the MI (the enhanced and the NF area combined) did not change significantly during the imaging period in either of the two models. Representative images including both EGE and LGE images are shown in Fig. 5.

Macroscopic and microscopic histopathologic evaluation of representative reperfused and non-reperfused MI is shown in Fig. 6. In the haematoxylin-eosin slides, widespread myocyte necrosis (nuclear shrinkage and nuclear loss), oedema (indicated by wide interstitium), and inflammatory cell infiltration (polymorphonuclear, considered neutrophils) and mononuclear (considered lymphocytes and macrophages) were observed in the central MI regions (corresponding to MRI NF areas) in both models. Using Prussian blue and Von Kossa staining, notable differences were detected between the reperfused and non-reperfused model. In reperfused MI, Prussian blue showed a relevant amount of iron deposits (extensive blue spots) throughout the whole MI area, but at the highest concentration in the border zone of the MI. Iron deposits were not detected at all in the core area of the nonreperfused MI. However, some iron-related blue spots were observed sporadically in the border zone of the nonreperfused MI. In reperfused MI, Von Kossa staining suggested that the periphery of the MI was strongly positive for calcium, while the inner core was negative. Calcium deposits were observed in the border zone of the nonreperfused MI as well, but the amount of the calcium

Table 2 Native T2 relaxation times ( $m s$, mean \pm SD)

\begin{tabular}{llll}
\hline & Remote & Infarct & No-flow region \\
\hline Reperfused & $57 \pm 5$ & $94 \pm 11^{*}$ & $56 \pm 14$ \\
Non-Reperfused & $63 \pm 7$ & $96 \pm 17^{*}$ & $60 \pm 9$ \\
$p$ & 0.326 & 0.765 & 0.432 \\
\hline
\end{tabular}

"Significant difference $(p<0.001)$ compared to the remote myocardium was clearly smaller and less widespread than in the reperfused MI.

\section{Discussion}

In this study we qualitatively investigated the MRI CA uptake characteristics of the NF area in non-reperfused and reperfused $\mathrm{MI}$ on day 4 after experimental induction of MI. Based on previous experience [15], we expected emergence of an NF area in the majority of the nonreperfused animals. The literature indicates that the NF is detected in human reperfused MI in around 68\% of patients [19] and our past experience of experiments in reperfused swine suggests that the NF can be expected in at least two thirds of animals. In this study, all reperfused animals presented with a significant NF on day 4 post MI.

Our study indicates that the NF size strongly depends on the time elapsed after the administration of the CA. Of note, with time the NF was able to take up the CA even in animals with non-reperfused MI. According to the current definition, the NF should be measured during the early CA kinetics [20]. Due to the significant CA uptake into the NF, this protocol should indeed be followed, as evaluating the NF 20-25 min later in the post-contrast period would result in notably larger enhanced MI regions and smaller NF areas. As previous reports highlight the prognostic value of the NF, its accurate quantification is even more relevant [21].

The reason for CA uptake in the reperfused animals may be explained by the observation that ischaemia/ reperfusion injury does not really cause total occlusion of the microvascular system [1]. Apparently, blood flow in the area of the early NF is not completely absent but rather very low [1]. The NF first appears centrally in the infarct core extending toward the epicardium over time. Myocardial blood flow progressively decreases within 2 to 3 hours after reperfusion, resulting in a twofold increase in the area of the NF. A further increase in NF size has been demonstrated up to 48 hours after initiation of reperfusion [1]. In contrast, myocardial blood flow in non- 


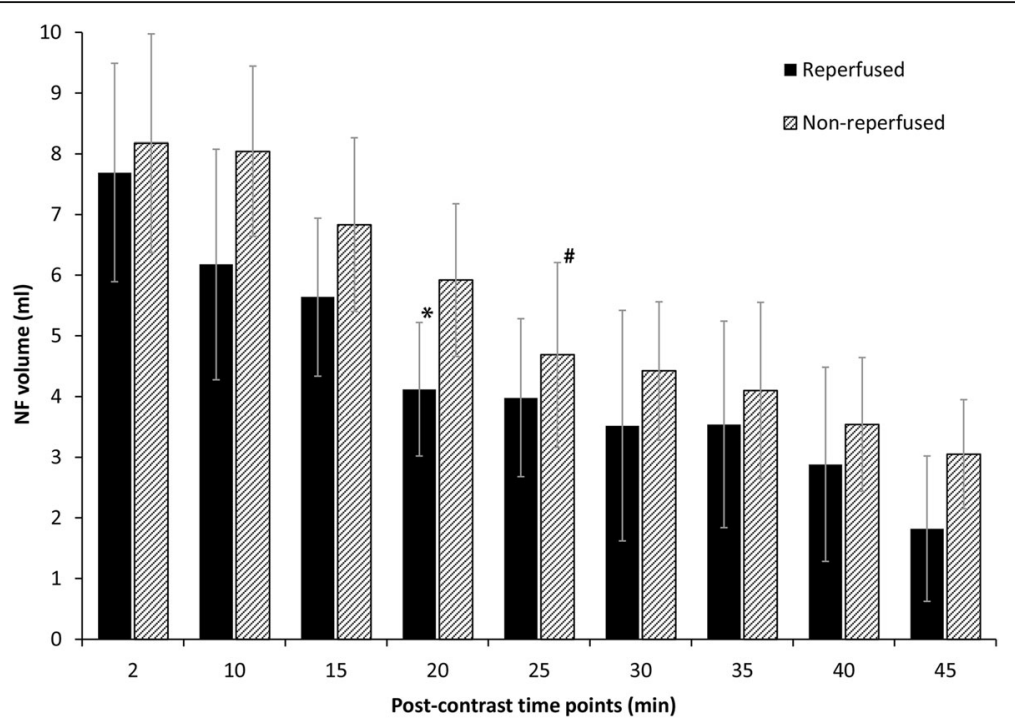

Fig. 4 Change over time in the no-flow region (NF) volume after contrast agent injection in reperfused and non-reperfused myocardial infarct. The first NF measurement performed at 2 min after contrast agent injection by early gadolinium enhancement imaging was considered the reference. *Significant

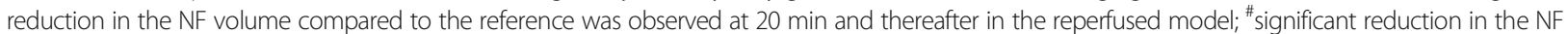
volume compared to the reference at $25 \mathrm{~min}$ and thereafter in the non-reperfused model

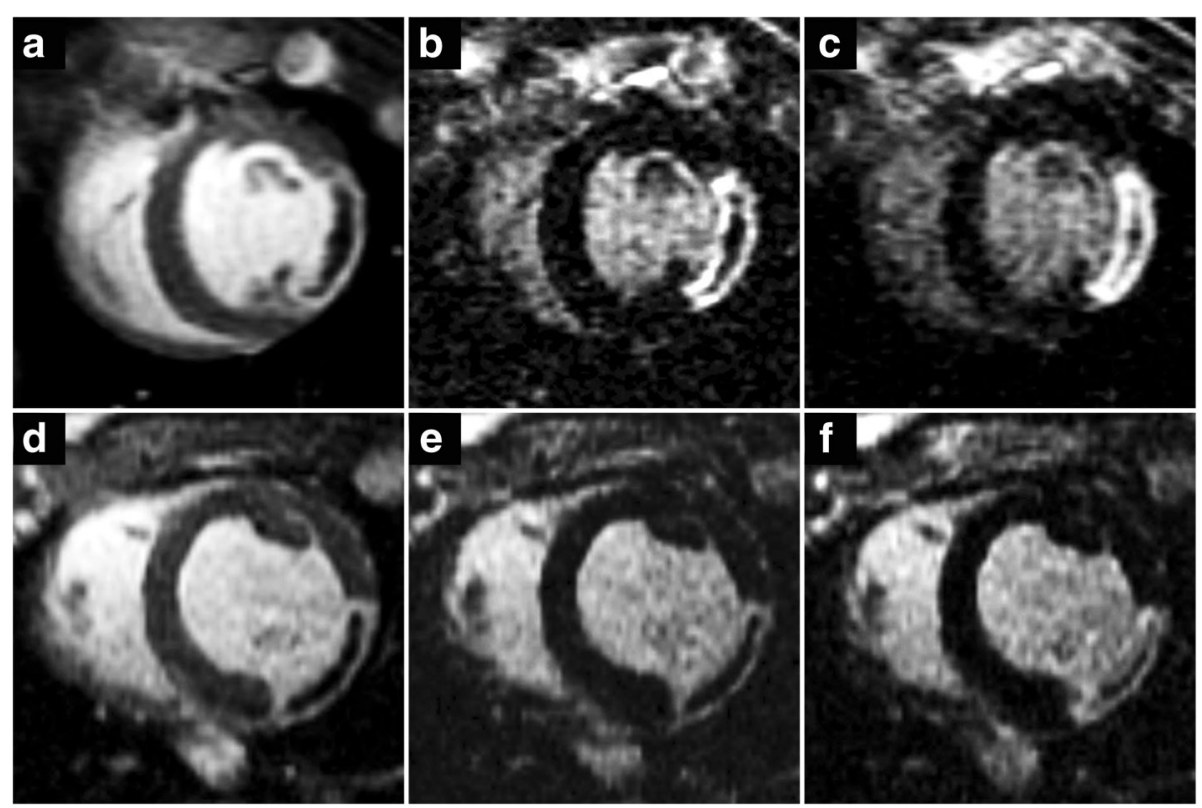

Fig. 5 Contrast uptake characteristics of the no-flow region (NF) in reperfused and non-reperfused myocardial infarct (MI). Short-axis oriented post-contrast inversion recovery images acquired in a reperfused (top row) and a non-reperfused (bottom row) animal at 2 min (a, d), 15 min $(\mathbf{b}, \mathbf{e})$, and $45 \mathrm{~min}(\mathbf{c}, \mathbf{f})$ after the administration of the contrast agent (CA). Early gadolinium enhancement images (a, d) are best to visualise the NF, while late gadolinium enhancement images at 15 min post contrast (b, e) are regularly used clinically to highlight MI. The area of the NF is the largest immediately after CA administration and gradually shrinks over time. By the end of the imaging session 45 min post contrast $(\mathbf{c}, \mathbf{f})$ the area previously shown as the NF now seems to be partially highlighted. CA distribution within the NF, however, is not uniform and the NF still contains unenhanced areas 


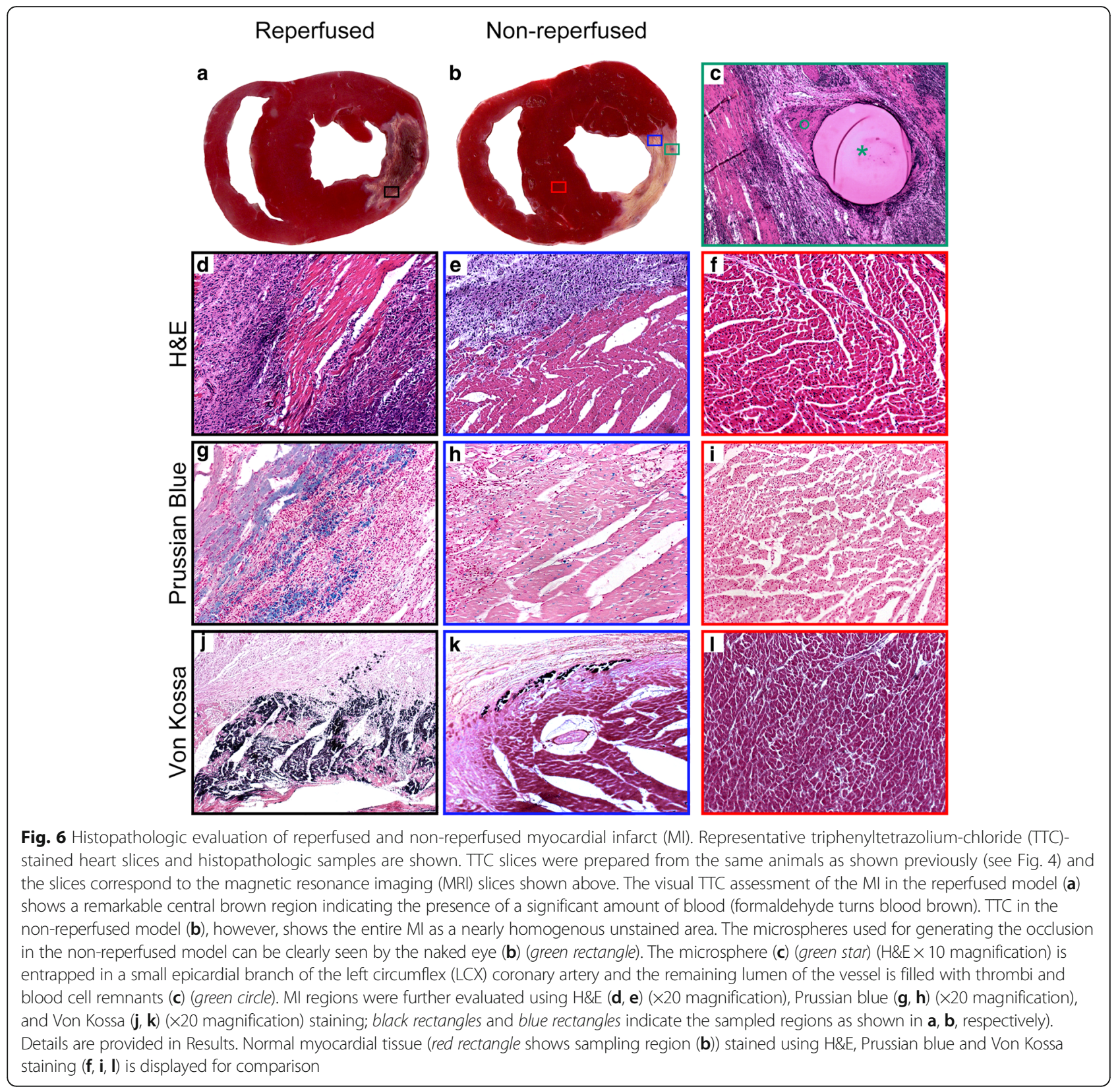

reperfused MI is completely absent. Nevertheless, as our results indicate, the NF area in non-reperfused MI also takes up CA, which has also been reported in prior studies.

Wang et al. have shown that the NF in nonreperfused MIs can be detected by EGE and LGE imaging up to 22-32 min after CA administration. It then disappears as the area defined as the NF on EGE images becomes enhanced [14]. Our study partially supports this observation, but it also indicates that the NF in non-reperfused MI can be detected beyond approximately $30 \mathrm{~min}$ and even at $45 \mathrm{~min}$ after the administration of CA.
Assuming total coronary artery occlusion in the non-reperfused MI model, the only possible mechanism for CA uptake is slow interstitial diffusion from the surrounding tissues that are still well-supplied [14]. The rim of the MI is highlighted even as early as the time of the EGE acquisition, presumably by CA from intact collaterals. This rim area of the MI is usually composed of a mixture of viable and nonviable myocytes [22]. The collateral circulation supplying this peri-infarct territory keeps the myocardial tissue partially alive, and serves as a CA source as well. The peri-infarct area, however, also contains vessels with damaged endothelial lining, allowing the accumulation 
of the CA in the extracellular space. This extracellular space may serve as a pool to supply the adjacent infarct area, including the NF, with CA. Interestingly, the reperfused and the non-reperfused MIs accumulate CA at a similar rate. The latter observation may confirm the hypothesis that most of the CA uptake is through slow interstitial diffusion in both MI models. If the blood flow is not completely absent in the reperfused case, as suggested by others [20], a vascular component may contribute to the access to CA. This flow, however, may be extremely limited, as the rate of contrast uptake in both models seems similar.

In this project we studied the presence of hemosiderin components by $\mathrm{T} 2$ relaxation rate measurements. Although $\mathrm{T} 2 *$ mapping is the most widely used technique for the detection of haemorrhage, T2 mapping has also been established for such assessment [23]. Our results indicate that $\mathrm{T} 2$ measured in the NF was not significantly different from the $\mathrm{T} 2$ observed in the remote myocardium and also did not differ between the models. Although this observation seems unexpected given that our histopathologic studies showed extensive hemosiderin deposition in the reperfused but not in the non-reperfused NF, it might be explained by the possible overlap of oedema (causing T2 increase) and haemorrhage (causing T2 decrease) resulting in an apparent net zero T2 change compared to normal tissue in the reperfused model. On the other hand, an absence of both oedema and haemorrhage would also result in an unchanged T2 in the non-reperfused model.

Another histopathologic difference between the two models was the presence of calcium deposits in the NF, which may also contribute to T2 shortening. Calcium deposition is a component of reperfusion injury [24], and its presence only in the reperfused animals further supports the successful total occlusion of the coronary arteries in the non-reperfused animals.

\section{Limitations}

The major limitation of this study is that the pulse sequence used to image the MI only allowed the qualitative assessment of the contrast uptake in the NF area. To quantitatively evaluate the diffusionrelated parameters to characterise the contrast uptake, fast $\mathrm{T} 1$ mapping sequences would be needed, as the speed of the conventional T1 mapping protocols is insufficient to monitor such diffusion changes. Novel modified Look-Locker inversion recovery (MOLLI)based pulse sequences [25] would allow for singleshot, single-breath-hold T1 mapping with high spatial and temporal resolution. However, such MOLLI pulse sequences were not available on the MRI scanner utilised for this study. In addition, post-contrast T1 mapping would have allowed the calculation of extracellular volume fraction providing additional details on myocardial tissue characterization. Postcontrast T1 mapping without a MOLLI sequence, however, would have required an extensive acquisition time, interfering with our study design, which required post-contrast imaging in 5-min intervals.

Another minor limitation is that the presented MRI data were obtained 4 days after induction of MI; thus, our results are not necessarily applicable to MI assessed at different time points. Also, the animal models used in this study may not be fully comparable to human MI. Thus, our results cannot be directly translated to human myocardial tissue characterization. Finally, the number of animals in this project was small but the study was still sufficiently powered with this cohort.

\section{Conclusions}

NF in non-reperfused and reperfused MIs have similar characteristics on MRI despite the different pathophysiologic and underlying histopathologic conditions, indicating that the presence of the NF alone cannot differentiate between these two types of MI.

\begin{abstract}
Abbreviations
CA: contrast agent; EGE: Early gadolinium enhancement; H\&E: Haematoxylin and eosin; IR: Inversion recovery; LCX: Left circumflex coronary artery; LGE: Late gadolinium enhancement; LV: Left ventricle; LVM: Left ventricular mass; MI: Myocardial infarct; MIF: Myocardial infarct fraction; MO: Microvascular obstruction; MRI: Magnetic resonance imaging; NF: No-flow region; NFF: No-flow region fraction; SD: Standard deviation; SI: Signal intensity; TE: Echo time; TR: Repetition time; TTC: Triphenyltetrazolium-chloride
\end{abstract}

\section{Funding}

This study was supported by a grant from the National Institutes of Health, National Heart, Lung and Blood Institute (Grant\# R42-HL084844 to AVS).

Availability of data and materials

The relevant data have been included in the manuscript.

\section{Authors' contributions}

GAE: study design, data interpretation, manuscript preparation. TS: study design, animal experiments, MRI studies, data interpretation, manuscript revision. RJvdG; data analysis, manuscript revision. PS: study design, data interpretation, manuscript revision. PPK: study design, animal experiments, MRI studies, manuscript revision. ZL: animal experiments, MRI studies, manuscript revision. RK: study design, animal experiments, MRI studies. DW: histopathology, data interpretation, manuscript revision. BCB: study design, animal model, manuscript revision. AVS: study design, animal model, MRI studies, manuscript drafting, revision. All authors read and approved the final manuscript.

\section{Competing interests}

Drs. Varga-Szemes, Kirschner, and Lenkey were employees, Dr. Simor is a consultant, and Dr. Elgavish is officer of Elgavish Paramagnetics Inc. Dr. Varga-Szemes is a consultant for and/or receives research support from Guerbet and Siemens. The other authors have no conflicts of interest.

\section{Publisher's Note}

Springer Nature remains neutral with regard to jurisdictional claims in published maps and institutional affiliations.

\section{Author details}

${ }^{1}$ Department of Biochemistry and Molecular Genetics, University of Alabama at Birmingham, MCLM 556, Birmingham, AL 35294-0005, USA. ²Division of 
Cardiovascular Disease, Department of Medicine, University of Alabama at Birmingham, FOT 907, Birmingham, AL 35294-3407, USA. ${ }^{3}$ Elgavish Paramagnetics Inc., 1737 Valpar Dr, Hoover, AL 35226, USA. ${ }^{4}$ Heart Institute, Medical School, University of Pecs, Szigeti ut 12, Pecs 7624, Hungary. ${ }^{5}$ Department of Radiology, Leiden University Medical Center, Albinusdreef 2, Leiden 2333 ZA, The Netherlands. ${ }^{6}$ Division of Cardiovascular Imaging, Department of Radiology and Radiological Science, Medical University of South Carolina, 25 Courtenay Dr, Charleston, SC 29425, USA. ${ }^{7}$ Department of Pathology, Histomorphometry and Molecular Analysis Core, University of Alabama at Birmingham, LHRB 589A, Birmingham, AL 35294-0007, USA.

\section{Received: 25 January 2017 Accepted: 8 March 2017}

Published online: 29 June 2017

\section{References}

1. Bekkers SC, Yazdani SK, Virmani R, Waltenberger J (2010) Microvascular obstruction: underlying pathophysiology and clinical diagnosis. J Am Coll Cardiol 55:1649-1660

2. Braunwald E, Kloner RA (1985) Myocardial reperfusion: a double-edged sword? J Clin Invest 76:1713-1719

3. Kloner RA, Ganote CE, Jennings RB (1974) The "no-reflow" phenomenon after temporary coronary occlusion in the dog. J Clin Invest 54:1496-1508

4. Nijveldt R, Hofman MB, Hirsch A et al (2009) Assessment of microvascular obstruction and prediction of short-term remodeling after acute myocardial infarction: cardiac MR imaging study. Radiology 250:363-370

5. Mewton N, Bonnefoy E, Revel D et al (2009) Presence and extent of cardiac magnetic resonance microvascular obstruction in reperfused non-ST-elevated myocardial infarction and correlation with infarct size and myocardial enzyme release. Cardiology 113:50-58

6. Mather AN, Lockie T, Nagel E et al (2009) Appearance of microvascular obstruction on high resolution first-pass perfusion, early and late gadolinium enhancement CMR in patients with acute myocardial infarction. J Cardiovasc Magn Reson 11:33

7. Bekkers SC, Backes WH, Kim RJ et al (2009) Detection and characteristics of microvascular obstruction in reperfused acute myocardial infarction using an optimized protocol for contrast-enhanced cardiovascular magnetic resonance imaging. Eur Radiol 19:2904-2912

8. Rochitte CE, Lima JAC, Bluemke DA et al (1998) Magnitude and time course of microvascular obstruction and tissue injury after acute myocardial infarction. Circulation 98:1006-1014

9. de Waha S, Desch S, Eitel I et al (2010) Impact of early vs. late microvascular obstruction assessed by magnetic resonance imaging on long-term outcome after ST-elevation myocardial infarction: a comparison with traditional prognostic markers. Eur Heart J 31:2660-2668

10. Orn S, Manhenke C, Greve OJ et al (2009) Microvascular obstruction is a major determinant of infarct healing and subsequent left ventricular remodelling following primary percutaneous coronary intervention. Eur Heart J 30:1978-1985

11. Wu KC, Zerhouni EA, Judd RM et al (1998) Prognostic significance of microvascular obstruction by magnetic resonance imaging in patients with acute myocardial infarction. Circulation 97:765-772

12. Hamirani YS, Wong A, Kramer CM, Salerno M (2014) Effect of microvascular obstruction and intramyocardial hemorrhage by CMR on LV remodeling and outcomes after myocardial infarction: a systematic review and metaanalysis. JACC Cardiovasc Imaging 7:940-952

13. Desch S, Eitel I, Schmitt J et al (2009) Effect of coronary collaterals on microvascular obstruction as assessed by magnetic resonance imaging in patients with acute ST-elevation myocardial infarction treated by primary coronary intervention. Am J Cardiol 104:1204-1209

14. Wang Y, Sun W, Cao G et al (2006) Delayed hyperenhancement patterns in occlusive and reperfused myocardial infarcts during different healing stages. J Magn Reson Imaging 24:851-857

15. Varga-Szemes A, Kiss P, Brott BC et al (2013) Embozene microspheres induced nonreperfused myocardial infarction in an experimental swine model. Catheter Cardiovasc Interv 81:689-697

16. Amado LC, Gerber BL, Gupta SN et al (2004) Accurate and objective infarct sizing by contrast-enhanced magnetic resonance imaging in a canine myocardial infarction model. J Am Coll Cardiol 44:2383-2389

17. Bondarenko O, Beek AM, Hofman MB et al (2005) Standardizing the definition of hyperenhancement in the quantitative assessment of infarct size and myocardial viability using delayed contrast-enhanced CMR. J Cardiovasc Magn Reson 7:481-485

18. Fishbein M, Meerbaum S, Rit J et al (1981) Early phase acute myocardial infarct size quantification: validation of the triphenyl tetrazolium chloride tissue enzyme staining technique. Am Heart J 101:593-600

19. de Waha S, Desch S, Eitel I et al (2012) Relationship and prognostic value of microvascular obstruction and infarct size in ST-elevation myocardial infarction as visualised by magnetic resonance imaging. Clin Res Cardiol 101:487-495

20. Wu KC (2012) CMR of microvascular obstruction and hemorrhage in myocardial infarction. J Cardiovasc Magn Reson 14:68

21. van Kranenburg M, Magro M, Thiele $\mathrm{H}$ et al (2014) Prognostic value of microvascular obstruction and infarct size, as measured by CMR in STEMI patients. JACC Cardiovasc Imaging 7:930-939

22. Varga-Szemes A, Simor T, Lenkey Z et al (2014) Infarct density distribution by MRI in the porcine model of acute and chronic myocardial infarction as a potential method transferable to the clinic. Int J Cardiovasc Imaging 30:937-948

23. Guo H, Au WY, Cheung JS et al (2009) Myocardial T2 quantitation in patients with iron overload at 3 Tesla. J Magn Reson Imaging 30:394-400

24. Saeed M, Hetts S, Wilson M (2010) Reperfusion injury components and manifestations determined by cardiovascular MR and MDCT imaging. World J Radiol 2:1-14

25. Kellman P, Hansen MS (2014) T1-mapping in the heart: accuracy and precision. J Cardiovasc Magn Reson 16:2

\section{Submit your manuscript to a SpringerOpen ${ }^{\circ}$ journal and benefit from:}

- Convenient online submission

- Rigorous peer review

- Immediate publication on acceptance

- Open access: articles freely available online

- High visibility within the field

- Retaining the copyright to your article

Submit your next manuscript at $>$ springeropen.com 